\title{
SAD Phasing for easy and challenging problems
}

\author{
Thomas C. Terwilliger $^{1}$ \\ ${ }^{1}$ Los Alamos National Laboratory, Los Alamos NM 87545 USA \\ E-mail: terwilliger@lanl.gov
}

A key step in SAD phasing is choosing how accurately the data need to be measured to have a good chance of success. In this workshiop we will describe tools for planning a SAD experiment, for evaluating your SAD data and for solving your structure by SAD phasing.

The phenix.plan_sad_experiment tool helps you identify the overall I/sigI needed in a dataset to find the substructure and obtain useful phase information. The phenix.scale_and_merge tool scales unmerged SAD data from one or more crystals using local scaling and optimizes the anomalous signal by identifying the systematic differences among datasets. The phenix.anomalous_signal tool estimates the anomalous signal in a dataset and estimates the probability that the dataset can be solved and the likely figure of merit of phasing, and the phenix.autosol tool will carry out automated structure solution from SAD data. These tools will be discussed and demonstrated using SAD datasets accessible to everyone in the workshop. 